\title{
A New Combinatoric Estimate for Cluster Expansions ${ }^{\star}$
}

\author{
Guy A. Battle III
}

Mathematics Department, Texas A\& M University, College Station, TX 77843, USA

\begin{abstract}
We state and prove a new and previously unsuspected tree graph inequality, which is significantly stronger than the one commonly applied to cluster expansions. The older inequality controls the counting problem in the convergence proof of such an expansion, but the new inequality does more: it also exhibits extra $1 / n$ ! factors that can be applied to the cancellation of number divergences. The proof of this new combinatoric estimate is completely elementary.
\end{abstract}

\section{Introduction}

For the past two years a new method of tree graph estimation has been applied to cluster expansions in statistical mechanics and quantum field theory [1-4]. The technique is based on a very powerful and flexible combinatoric identity that first appeared in [1]. However, the method has been slow to attract attention - in part, because the combinatoric identity has to be supplemented with additional topological ordering concepts if one wishes to apply it to the problem of cancelling the number divergence appearing in a cluster expansion. The goal of this paper is to prove a new and different combinatoric estimate whose application is equally powerful but does not require these auxiliary notions.

For convenience we repeat the basic definitions given in [1].

Definition 1.1. Let $\left(S, p_{1}\right)$ be a pointed set with cardinality $N$. An ordered connectivity graph (o.c.g.) on $\left(S, p_{1}\right)$ is a mapping $G$ from $\{2, \ldots, N\}$ into $S \times S$ such that if $G(i)=\left(g_{1}(i), g_{2}(i)\right)$, then

(a) $g_{2}(i) \neq g_{2}(j)$ for $i \neq j$,

(b) $g_{1}(i) \in\left\{p_{1}\right\} \cup\left\{g_{2}(j) \mid j<i\right\}$.

Definition 1.2. A tree graph of order $N$ is a mapping $\eta$ from $\{2, \ldots, N\}$ into $\{1, \ldots, N-1\}$ such that $\eta(i)<i$.

* Supported in part by the National Science Foundation under Grant No. MCS-8301116 
For each o.c.g. $G$ on $\left(S, p_{1}\right)$ we can associate a canonical tree graph $\eta_{G}$ of order $N$ : we define it by

$$
\eta_{G}(i)=g_{2}^{-1}\left(g_{1}(i)\right)
$$

with the convention that $g_{2}(1)=p_{1}$.

Definition 1.3. An unordered connectivity graph (u.c.g.) on $\left(S, p_{1}\right)$ is the range of some o.c.g. on $\left(S, p_{1}\right)$.

For a given o.c.g. $G$ on $\left(S, p_{1}\right)$ we denote the u.c.g. arising from it by $T_{G}$.

Now consider the following monomials in $s_{1}, \ldots, s_{N-1}$ :

$$
f(\eta ; s)=\prod_{i=2}^{N}\left(s_{\eta(i)} \ldots s_{i-2}\right) .
$$

The combinatoric estimate that has been applied in the past to the estimation of cluster expansions (see, e.g., [5]) is well known:

$$
\sum_{|\eta|=N} \prod_{i=1}^{N-1}\left(\int_{0}^{1} d s_{i}\right) f(\eta ; s) \leqq e^{N},
$$

where $|\eta|$ denotes the order of $\eta$. The combinatoric identity introduced in [1] turns out to be significantly more powerful:

$$
\sum_{G: T_{G}=T} \prod_{i=1}^{N-1}\left(\int_{0}^{1} d s_{i}\right) f_{G}(s)=1,
$$

where $f_{G}(s)=f\left(\eta_{G} ; s\right)$. The usefulness of this identity was further advertized in [6]. We should mention in passing that one can combine (1.4) with Cayley's theorem [7] to replace (1.3) with an identity:

$$
\sum_{|\eta|=N} \prod_{i=1}^{N-1}\left(\int_{0}^{1} d s_{i}\right) f(\eta ; s)=\frac{N^{N-1}}{N !} .
$$

The proof is due to R. W. Robinson and is given in [1].

Our aim is to prove the following result:

Theorem 1.4. For a given tree graph $\eta$ of order $N$, let $d_{\eta}(i)$ denote the cardinality of $\eta^{-1}(\{i\})$. Then

$$
\sum_{|\eta|=N}\left(\prod_{i=1}^{N-1} d_{\eta}(i) !\right) \prod_{i=1}^{N-1}\left(\int_{0}^{1} d s_{i}\right) f(\eta ; s) \leqq 4^{N} .
$$

Inequality (1.6) is obviously much stronger than (1.3). With regard to applications, there are two important remarks to be made. First the application of (1.6) to cluster expansions will yield the same new results as the application of (1.4) already has in [1-4]. Second, (1.6) can be applied in the same simple manner that (1.3) has always been applied - with the counting problem automatically solved by a supremum over tree graphs. Compare this to the more subtle counting scheme demanded by the application of (1.4) and the partial re-introduction of order to the unordered connectivity graphs. The point is that (1.6) enables us to dispense with the concepts of representation two and representation three [1-4]. 
When informed of the above result, Tom Kennedy immediately noticed that a $c^{N}$ bound on the sum in (1.6) follows from the result (9) in [6]. His argument is delightfully brief, and we include it as an appendix. The catch to this, of course, is that the result (9) in [6] is not so easy to prove: the argument combines (1.4) above with the notions of representations two and three. (The outline given in [6] is extremely terse and refers heavily to [1] without mentioning the notion of representation three at all. A more expository description of the argument will appear in [8].) Still, it is interesting that a bound like (1.6) follows from (1.4). We should also mention that Kennedy (private communication) has an alternative proof of $(1.4) \Rightarrow((9)$ in [6]); it is more combinatoric in nature and does not use representation three.

We prove our theorem from scratch - i.e., we do not assume (1.4) at all. Indeed, the notion of tree graph is the only notion used; o.c.g.'s and u.c.g.'s are not needed. The key to our theorem is the following identity:

Lemma 1.5. For a given tree graph $\eta$ of order $N$, let $\pi(\eta)$ denote the number of permutations $P$ of $\{2, \ldots, N\}$ for which $\eta \circ P$ is still a tree graph. Then

$$
\prod_{i=1}^{N-1}\left(\int_{0}^{1} d s_{i}\right) f(\eta ; s)=\frac{1}{\pi(\eta)}
$$

This identity is stated in [1] without proof and without application. (At that time it was just a combinatoric curiosity that had been incidentally discovered.) We will give an elementary proof in Sect. 2.

Proof of Theorem 1.4. Clearly, $\prod_{i=1}^{N-1} d_{\eta}(i)$ ! isthe number of permutations $P$ forwhich $\eta \circ P=\eta$. Hence

$$
\pi(\eta)=\sum_{\psi \in A_{\eta}} \prod_{i=1}^{N-1} d_{\psi}(i) !
$$

where $A_{\eta}$ is the set of all tree graphs $\psi$ for which $\psi=\eta \circ P$ for some permutation $P$. Bur for such $\psi$ it is obvious that $d_{\psi}(i)=d_{\eta}(i)$, so (1.8) becomes

$$
\pi(\eta)=\left|A_{\eta}\right| \prod_{i=1}^{N-1} d_{\eta}(i) !
$$

It follows from Lemma 1.5 that

$$
\sum_{|\eta|=N}\left(\prod_{i=1}^{N-1} d_{\eta}(i) !\right) \prod_{i=1}^{N-1}\left(\int_{0}^{1} d s_{i}\right) f(\eta ; s)=\sum_{|\eta|=N} \frac{1}{\left|\mathrm{~A}_{\eta}\right|} .
$$

Let $\mathscr{P}$ be the set of sets $A_{\eta}$; since $\mathscr{P}$ partitions the set of tree graphs, we have

$$
\begin{aligned}
\sum_{|\eta|=N} \frac{1}{\left|A_{\eta}\right|} & =\sum_{A \in \mathscr{P}} \sum_{\psi \in A} \frac{1}{|A|} \\
& =\sum_{A \in \mathscr{P}} 1 \\
& =|\mathscr{P}|,
\end{aligned}
$$


so we need only show that $|\mathscr{P}| \leqq 4^{N}$. Now we have already noted that $d_{\psi}(i)=d_{\eta}(i)$ for $\psi \in A_{\eta}$. The converse is also true: if $d_{\psi}(i)=d_{\eta}(i)$, then we can choose a permutation $P$ that maps each $\psi^{-1}(\{i\})$ one-to-one and onto $\eta^{-1}(\{i\})$, in which case $\psi=\eta \circ P$. Thus, $A_{\eta}$ is uniquely determined by $d_{\eta}$, and so we can define a mapping $\Gamma$ from

$$
\left\{\left(M_{1}, \ldots, M_{N-1}\right) \mid M_{i} \text { is a nonnegative integer and } \sum_{i=1}^{N-1} M_{i}=N-1\right\}
$$

into $\mathscr{P} \cup\{\phi\}$ by letting $\Gamma\left(M_{1}, \ldots, M_{N-1}\right)$ be that $A_{\eta}$ for which $d_{\eta}(i)=M_{i}$, with the convention that $\Gamma\left(M_{1}, \ldots, M_{N-1}\right)=\phi$ if there is no such tree graph $\eta$. Since the mapping is clearly onto, it follows that $|\mathscr{P}|$ is bounded by the cardinality of (1.12). On the other hand, it is a standard combinatoric fact that the cardinality of (1.12) is bounded by $4^{N}$.

\section{Proof of the Lemma}

Clearly, (1.2) can be written as

$$
f(\eta ; s)=\prod_{k=1}^{N-2} s_{k}^{N(k)}
$$

where $N(k)$ is the cardinality of

$$
\{i \mid \eta(i) \leqq k \leqq i-2\} .
$$

Hence

$$
\prod_{i=1}^{N-1}\left(\int_{0}^{1} d s_{i}\right) f(\eta ; s)=\prod_{k=1}^{N-2} \frac{1}{N(k)+1},
$$

so it suffices to show that

$$
\pi(\eta)=\prod_{k=1}^{N-2}(N(k)+1)
$$

For convenience we define $F(k)$ as the cardinality of

$$
\{i \mid \eta(i) \leqq k\} .
$$

Since $\eta(i)<i$, we have the inclusion

$$
\{i \mid 2 \leqq i \leqq k+1\} \subset\{i \mid \eta(i) \leqq k\},
$$

and therefore the equality of (2.5) to the disjoint union

$$
\{i \mid 2 \leqq i \leqq k+1\} \cup\{i \mid \eta(i) \leqq k<i-1\} .
$$

But the latter set is just (2.2), so

$$
F(k)=k+N(k) .
$$

Let $k_{0}=\sup \{\eta(i) \mid 2 \leqq i \leqq N\}$ : clearly,

$$
d_{\eta}\left(k_{0}\right)=N-1-F\left(k_{0}-1\right) .
$$


We are interested in all permutations $P$ for which $\eta \circ P$ is still a tree graph (i.e., $\eta(P(i))<i)$, so we are free to map the elements of $\eta^{-1}\left(\left\{k_{0}\right\}\right)$ one-to-one into $\left\{i \mid k_{0}<i \leqq N\right\}$. Thus, the $\eta(i)=k_{0}$ classification of the permutations is an $\left(N-k_{0}\right)$ choose- $\bar{d}_{\eta}\left(k_{0}\right)$ situation, where permutations of the subset are included, so we have split the set of allowed permutations into

$$
d_{\eta}\left(k_{0}\right) !\left(\begin{array}{c}
N-k_{0} \\
d_{\eta}\left(k_{0}\right)
\end{array}\right)=\frac{\left(N-k_{0}\right) !}{\left(F\left(k_{0}-1\right)-k_{0}+1\right) !}
$$

classes [where (2.9) has been applied].

Now consider a class (i.e., fix the permutation at the $\eta(i)=k_{0}$ level) and look at the possibilities for the $\eta(i)=k_{0}-1$ level: clearly,

$$
d_{\eta}\left(k_{0}-1\right)=F\left(k_{0}-1\right)-F\left(k_{0}-2\right) \text {. }
$$

We are free to map $\eta^{-1}\left(\left\{k_{0}-1\right\}\right)$ one-to-one into the subset of $\left\{i \mid k_{0}-1<i \leqq N\right\}$ complementing the subset already determined by the choice made at the $\eta(i)=k_{0}$ level. Since the latter subset has $d_{\eta}\left(k_{0}\right)$ elements, it follows from (2.9) that the former subset has

$$
N-k_{0}+1-d_{\eta}\left(k_{0}\right)=F\left(k_{0}-1\right)-k_{0}+2
$$

elements, so we have split our class of permutations into

$$
d_{\eta}\left(k_{0}-1\right) !\left(\begin{array}{c}
F\left(k_{0}-1\right)-k_{0}+2 \\
d_{\eta}\left(k_{0}-1\right)
\end{array}\right)=\frac{\left(F\left(k_{0}-1\right)-k_{0}+2\right) !}{\left(F\left(k_{0}-2\right)-k_{0}+2\right) !}
$$

sub-classes (where (2.11) has been applied).

Continuing this process, we see that at the $\eta(i)=k_{0}-k$ level (where the permutation is fixed at all higher levels) we are free to map $\eta^{-1}\left(\left\{k_{0}-k\right\}\right)$ one-toone into a pre-determined subset of $\left\{i \mid k_{0}-k<i \leqq N\right\}$ that has cardinality

$$
N-k_{0}+k-\sum_{\ell=0}^{k-1} d_{\eta}\left(k_{0}-\ell\right) \text {. }
$$

But the summation is just the cardinality of $\left\{i \mid \eta(i)>k_{0}-k\right\}$, which is obviously $N-1-F\left(k_{0}-k\right)$. Hence

$$
(2.14)=F\left(k_{0}-k\right)-k_{0}+k+1,
$$

so we have split our $\operatorname{sub}^{k-1}$-class into

$$
d_{\eta}\left(k_{0}-k\right) !\left(\begin{array}{c}
F\left(k_{0}-k\right)-k_{0}+k+1 \\
d_{\eta}\left(k_{0}-k\right)
\end{array}\right)
$$

$\mathrm{sub}^{k}$-classes. On the other hand,

$$
d_{\eta}\left(k_{0}-k\right)=F\left(k_{0}-k\right)-F\left(k_{0}-k-1\right),
$$

so (2.16) can be written as

$$
\frac{\left(F\left(k_{0}-k\right)-k_{0}+k+1\right) !}{\left(F\left(k_{0}-k-1\right)-k_{0}+k+1\right) !} .
$$


In summary, the total number of permutations $P$ for which $\eta \circ P$ is still a tree graph is given by

$$
\pi(\eta)=\frac{\left(N-k_{0}\right) !}{\left(F\left(k_{0}-1\right)-k_{0}+1\right) !} \prod_{k=1}^{k_{0}-1} \frac{\left(F\left(k_{0}-k\right)-k_{0}+k+1\right) !}{\left(F\left(k_{0}-k-1\right)-k_{0}+k+1\right) !},
$$

which obviously telescopes into

$$
\pi(\eta)=\left(N-k_{0}\right) ! \prod_{k=1}^{k_{0}-1}\left(F\left(k_{0}-k\right)-k_{0}+k+1\right) .
$$

Applying (2.8), we see that

$$
\begin{aligned}
\pi(\eta) & =\left(N-k_{0}\right) ! \prod_{\substack{k_{0}-1 \\
k_{0}-1}}^{k_{0}-1}\left(N\left(k_{0}-k\right)+1\right) \\
& =\left(N-k_{0}\right) ! \prod_{k=1}(N(k)+1)
\end{aligned}
$$

Since $k_{0}$ is the maximum value of $\eta$, we know that for $k \geqq k_{0} F(k)=N-1$, and therefore $N-k=N(k)+1$. Thus

$$
\left(N-k_{0}\right) !=\prod_{k=k_{0}}^{N-2}(N(k)+1)
$$

and so we finally have (2.4).

\section{Appendix}

Kennedy's observation that the result (9) in [6] implies

$$
\sum_{|\eta|=N} \prod_{i}\left(d_{\eta}(i) !\right) \prod_{i}\left(\int_{0}^{1} d s_{i}\right) f(\eta ; s) \leqq c^{N}
$$

is worth presenting because it is very brief and relates this paper to [6]. The essence of (9) in [6] is the inequality

$$
\begin{aligned}
& \sum_{X} \sum_{|\eta|=N} \prod_{i} C(X(i), X(\eta(i))) \prod_{i}\left(d_{\eta}(i) !\right) \prod_{i}\left(\int_{0}^{1} d s_{i}\right) f(\eta ; s) \\
& \leqq 4^{N}\left(\sup _{y \in A} \sum_{x \in A} C(x, y)\right)^{N-1}
\end{aligned}
$$

where $C$ is a positive function on $A \times A$ and $X$ ranges over one-to-one mappings of $\{1, \ldots, N\}$ into $A$ with $X(1)$ fixed. Now pick $|A|=N$ and $C(x, y)=1$ : the inequality becomes

$$
\sum_{X} \sum_{|\eta|=N} \prod_{i}\left(d_{\eta}(i) !\right) \prod_{i}\left(\int_{0}^{1} d s_{i}\right) f(\eta ; s) \leqq 4^{N} N^{N-1} .
$$

Since there are $(N-1)$ ! mappings $X$ in this case and the summand no longer depends on $X$, we have

$$
\sum_{|\eta|=N} \prod_{i}\left(d_{\eta}(i) !\right) \prod_{i}\left(\int_{0}^{1} d s_{i}\right) f(\eta ; s) \leqq \frac{4^{N} N^{N-1}}{(N-1) !} \leqq(4 e)^{N}
$$


Acknowledgements. I would like to thank Paul Federbush, who encouraged me to try finding a less mysterious way to realize the extra $1 / n$ ! factors that he discovered in cluster expansions two years ago. The application of our combinatoric identity to providing convergence of expansions was certainly powerful and elegant, but admittedly subtle. I would also like to thank Tom Kennedy for constructive comments on this result.

\section{References}

1. Battle, G., Federbush, P.: A phase cell cluster expansion for Euclidean field theories. Ann. Phys. 142, 95-139 (1982)

2. Battle, G., Federbush, P.: A phase cell cluster expansion for a hierarchical $\phi_{3}^{4}$ model. Commun. Math. Phys. 88, 263-293 (1983)

3. Battle, G.: Federbush mode decomposition of $\phi_{2}^{4}$. Preprint

4. Federbush, P.: Surface effects in Debye screening, a model example. Part I. Preprint

5. Glimm, J., Jaffe, A.: Quantum physics: a functional integral point of view. Berlin, Heidelberg, New York: Springer 1981

6. Battle, G., Federbush, P.: A note on cluster expansions, tree graph identities, extra $1 / N$ ! factors!!!. Lett. Math. Phys. 8, 55-57 (1984)

7. Harary, F., Palmer, E.M.: Graphical enumeration. New York: Academic Press 1973

8. Battle, G., Federbush, P., Robinson, R.: In preparation

Communicated by K. Osterwalder

Received November 28, 1983; in revised form March 2, 1984 
